\title{
Radioanalytical Chemistry for Automated Nuclear Waste Process Monitoring
}

(Project Number: 81923)

\section{Principal Investigator}

Oleg B. Egorov

Pacific Northwest National Laboratory

P.O. Box 999, MSIN P7-22

Richland, WA 99352

509-376-3485 (phone)

509-372-2156 (fax)

oleg.egorov@pnl.gov

\section{Co-Investigator}

Jay W. Grate

Pacific Northwest National Laboratory

P.O. Box 999, MSIN K8-93

Richland, WA 99352

509-376-4242 (phone)

509-376-5106 (fax)

jwgrate@pnl.gov

\section{Co-Principal Investigator}

Timothy A. DeVol

Environmental Engineering \& Science

Clemson University

Clemson, SC 29634-0919

864-656-1014 (phone)

tim.devol@ces.clemson.edu

Graduate Students Actively Involved in the Project: 3

Undergraduate Students Involved (Part-Time) in the Project: 3 


\section{Research Objective}

This research is directed toward rapid, sensitive, and selective determination of beta- and alphaemitting radionuclides such as ${ }^{99} \mathrm{Tc},{ }^{90} \mathrm{Sr}$, and transuranium (TRU) elements in low-activity waste (LAW) processing streams. The overall technical approach is based on automated radiochemical measurement principles. Nuclear waste process streams are particularly challenging due to the complex, high-ionic-strength, caustic brine sample matrix, the presence of interfering radionuclides, and the sometimes variable and uncertain speciation of the radionuclides to be analyzed. As a result, matrix modification, speciation control, and separation chemistries are required for use in automated process analyzers. Significant knowledge gaps exist relative to designing chemistries for such analyzers so that radionuclides can be quantitatively and rapidly separated and analyzed in high ionic strength solutions derived from low-activity waste processing operations. This research is addressing these knowledge gaps and automated microscale fluid handling techniques will be used to integrate sample modification, chemical separation chemistries, and radiometric detection steps within a single functional process analytical instrument.

The outcome of these investigations will be the knowledge necessary to choose appropriate chemistries for sample matrix modification and analyte speciation control and chemistries for rapid and selective separation and preconcentration of target radionuclides from complex sample matrices. In addition, new approaches for quantification of alpha emitters in solution using solid-state diode detectors, as well as improved instrumentation and signal processing techniques for use with solid-state and scintillation detectors, will be developed. New knowledge of the performance of separation materials, matrix modification and speciation control chemistries, instrument configurations, and quantitative analytical approaches will provide the basis for designing effective instrumentation for radioanalytical process monitoring. Specific analytical targets include ${ }^{99} \mathrm{Tc},{ }^{90} \mathrm{Sr}$, and TRU actinides.

\section{Research Progress and Implications}

This report summarizes work as of 8 months into a 3-year program. Because of the immediate relevance to our applied effort in the development of the on-line ${ }^{99} \mathrm{Tc}$ process monitor for the Waste Treatment Plant at Hanford, our initial research effort has been directed at 1) chemistry for rapid, automated ${ }^{99} \mathrm{Tc}$ speciation control; 2) ${ }^{99} \mathrm{Tc}$ separation chemistry; and 3) radioanalytical chemistry of the Hanford waste. In the area of radiation detection, our effort to date has been targeted toward the investigation of state-of-the-art photo detectors and procurement of instrumentation needed for the project. 
In order to enable total ${ }^{99} \mathrm{Tc}$ analysis in process solutions, oxidation chemistries and automated procedures must be developed that convert all of the Tc species to pertechnetate. For the implementation in a process-monitor instrument, oxidation procedures must be rapid, reliable, and quantitative. In addition, oxidizing reagents must be stable over long periods of time. To enable rapid and reliable oxidation of the non-pertechnetate species to pertechnetate, we selected oxidation chemistry based on peroxidisulfate. Peroxidisulfate chemistry was selected and evaluated in detail using actual high-organic Hanford wastes with up to $70 \%$ content of the nonpertechnetate fraction. The oxidation chemistry was studied using an automated microwaveassisted reaction module. Our studies with actual waste indicated that sample acidification is necessary prior to oxidation of non-pertechnetate to remove copious quantities of nitrite that interferes with oxidation. Furthermore, we established that acidification and microwave heat treatment alone were not sufficient to enable rapid and quantitative oxidation. However, rapid and reliable oxidation of Tc was possible using peroxidisulfate reagent. Using microwave sample treatment, the oxidation was complete within 20 seconds. No catalyst was necessary to enable rapid and reliable oxidation. In addition, it is significant that complete destruction of the organic matter was determined to be unnecessary for the quantitative oxidation of nonpertechnetate species to pertechnetate. Consequently significant excess of the oxidant is not required for quantitative oxidation of non-pertechnetate. We evaluated the decomposition rate of peroxidisulfate reagent. Results indicate a reliable shelf life of 2 months in de-ionized water is feasible. Slower rates of decomposition and improved shelf life were obtained in slightly alkaline solutions ( $\mathrm{pH}$ 9). Our results to date indicate that peroxidisulfate oxidation chemistry represents the chemistry of choice for rapid and reliable Tc speciation control in aged waste samples; the chemistry enables rapid and reliable oxidation of non-pertechnetate.

We continue detailed development and investigation of the column separation chemistries for separating ${ }^{99} \mathrm{Tc}$ (VII) from radioactive interferences in aged waste. The separation format selected for detailed investigation is based on the use of strongly basic anion exchange sorbent material. Our results to date indicate that rapid and selective separation of ${ }^{99} \mathrm{Tc}$ from major radioactive interferents and stable matrix is possible. Nevertheless, a sequence of column wash steps is required to ensure adequate removal of the interfering species required for accurate ${ }^{99} \mathrm{Tc}$ determination in the aged waste. Using anion exchange chromatographic material, separation from major radioactive species (e.g., ${ }^{90} \mathrm{Sr} /{ }^{90} \mathrm{Y},{ }^{137} \mathrm{Cs}$ ) is straightforward. However, this format is more challenging for separating Tc from other anionic species. Experiments with actual Hanford wastes indicated that Tc separation from $\mathrm{Sn}, \mathrm{Sb}, \mathrm{Ru}$, and $\mathrm{Rh}$ is required to enable accurate analysis of ${ }^{99} \mathrm{Tc}$ under process-monitoring conditions. Work in progress is directed at the development of elution sequences to address these separation requirements.

We continue to investigate the trade-offs of the various photodetectors. We have purchased an avalanche photodiode detector package for evaluation relative to the photomultiplier tube (PMT). Initial results indicate that for high-level waste process-monitoring applications, the PMT may be 
the best suited, as compact size and signal-to-noise ratio are not the major considerations. Digital signal processing of the electronic pulses from a radiation detector is becoming mainstream. We learned of the power and limitations associated with the use of a softwarebased digital signal processing system under another EMSP project (No. 70179). The primary limitation imposed by a software-based approach is the maximum count rate, which is considerably more limiting for the quantification of high-level waste process streams than for environmental radiation measurements. After considerable investigation, a Digital Gamma Finder - 4C (DGF-4C, X-ray Instrumentation Associates) was purchased for the project. The DGF-4C offers the possibility of hardware-implemented real-time digital signal processing. The negative output pulses from the photodetectors will be digitized at a rate of $40 \mathrm{MHz}$ with 12-bit precision using the XIA's DGF-4C digital spectrometer and waveform digitizer. All four channels of the DGF-4C will be used to digitize anode and dynode pulses from two photodetectors. The DGF-4C module was housed in a low-noise CAMAC crate. Communication to the DGF-4C module is through a Jorway 73A SCSI crate controller to a personal computer.

Standard DGF-4C control software, developed at XIA, will be used initially to collect and analyze the data. As needed, the data can be saved as list mode to the personal computer hard disk and analyzed off-line with the DGF Viewer. The DGF Viewer runs under an interactive programming and data analysis environment IGOR Pro (Wavemetrics, Inc.) whose programming language and graphics were used to analyze the data offline. This gives us the flexibility to optimize the data acquisition algorithms before they are programmed into the fieldprogrammable gate arrays of the DGF-4C. The DGF-4C, CAMAC crate, Jorway 73A Fast CAMAC controller and SCSI card for the PC were received by April 2002. We are in the early stages of the instrument development and software evaluation.

\section{Planned Activities}

During the remainder of the first year, we will continue investigation, developing, testing of the

${ }^{99} \mathrm{Tc}$ separation chemistries for rapid, automated radiochemical analysis of ${ }^{99} \mathrm{Tc}$ in Hanford waste-processing streams. We have held discussions with Savannah River Site personnel on the needs of Sr and TRU monitoring for the Salt Processing Facility. Savannah River Site needs are being used to direct our research plans for the second and third years of this project, which will be directed at automated monitoring of ${ }^{90} \mathrm{Sr}$ and TRU in aged nuclear waste streams.

\section{Information Access}

Egorov O, MO'Hara, and J Grate. 2002. Automation of the radiochemical analysis: From groundwater monitoring to nuclear waste analysis. Abstracts of Papers, 223rd ACS National Meeting, April 7-11, 2002, Orlando, Florida. 
Egorov O, M O'Hara, and J Grate. 2002. "Automated radiochemical analysis of total Tc-99 in nuclear waste processing streams." Abstracts of Papers, 223rd ACS National Meeting, April 7-11, 2002, Orlando, Florida.

Egorov O; T DeVol, and J Grate. 2001. "Advances in automated radioanalytical chemistry: From groundwater monitoring to nuclear waste analysis." Abstracts of Papers, 222nd ACS National Meeting, August 26-30, 2001, Chicago, Illinois. 